\title{
Recovering a fiber Bragg grating axial strain distribution from its reflection spectrum
}

\author{
L. Rodriguez-Cobo, ${ }^{*}$ A. Cobo, and J. M. Lopez-Higuera \\ Photonics Engineering Group, University of Cantabria, Av. Los Castros, Santander 39005, Spain \\ *Corresponding author: luis.rodriguez@unican.es
}

Received March 25, 2013; revised May 6, 2013; accepted May 27, 2013;

posted June 3, 2013 (Doc. ID 187775); published June 26, 2013

\begin{abstract}
A processing scheme able to obtain any arbitrary intragrating strain distribution of a fiber Bragg grating (FBG) is proposed and demonstrated. The processing method employs just the intensity of the FBG reflection spectrum to obtain its deformation profile by combining a geometrical processing scheme with the particle swarm optimization technique. The technique has been evaluated using several spectra generated from very heterogeneous strain distributions and with a real spectrum obtained from a $5 \mathrm{~mm}$ length FBG under an asymmetric perturbation. The achieved results exhibit a good spatial resolution $(\Delta \approx 0.5 \mathrm{~mm})$ and repeatability. (C) 2013 Optical Society of America

OCIS codes: (060.3735) Fiber Bragg gratings; (280.4788) Optical sensing and sensors.

http://dx.doi.org/10.1364/OL.38.002327
\end{abstract}

Fiber Bragg grating based transducers (FBGTs) [1] are a mature technology widely applied to monitor different materials and structures. Although in most of the FBGT applications the reported measurand is the integrated value along the FBG length [2], the axial measurand distribution inside the FBG structure is required for many others. Several approaches have been proposed to obtain the deformation profile along the FBG structure, but most of them require phase measurements of the reflection spectrum [3,4] or a priori knowledge of the strain distribution [5-7] . Another approach based on a computational method [ 8 ] has been proposed to retrieve the strain distribution of sampled FBGs just from their reflection spectra; however, the spatial resolution of this method is limited by the sampling period of the sampled FBG.

In this Letter, the particle swarm optimization (PSO) technique $[\underline{9}, \underline{10}]$ has been combined with a geometrical processing method to obtain any arbitrary axial strain profile of an FBG from its reflection spectrum. The geometrical processing is implemented as an error function that compares the desired spectrum (associated with the FBG to be measured) to different synthesized spectra [11] generated from the PSO candidate strain profiles. FBG length is divided into $N$ sections whose strain values are modified (PSO candidates) until measured and synthetic spectra match. Since an FBG under a nonuniform strain profile can generate very different spectra, the error function employed as minimization target must deal with these spectral variations. The whole technique (geometric processing and PSO) has been applied to different simulated scenarios to analyze its performance. Besides, a uniform FBG of $L=5 \mathrm{~mm}$ has been subjected to an asymmetric thermal perturbation and its reflection spectrum has been analyzed with the proposed technique. The achieved results exhibit a very good spatial resolution $(\Delta L=0.5 \mathrm{~mm})$, being mainly limited by the computation time.

The main problem when there is a lack of a priori knowledge about the strain distribution applied to an FBG is the wide divergence of the response spectra. The large amount of possible spectral shapes makes it difficult to compare between them in an efficient way. Previous related works [8] limit the FBG structure to make easier the comparison task between two spectra by ensuring some spectral artifacts that can be evaluated (i.e., several peaks for the sampled FBGs). The geometrical processing scheme must have enough sensitivity to distinguish between two close spectra and also enough goodness to assign a low error to a synthetic spectrum generated from a strain profile with less spatial resolution than the desired one (usually a continuous profile). To achieve these two goals the proposed metric analyzes several parameters related to the general "shape" of each spectrum. Each peak of the spectrum is associated with its two-dimensional coordinates (wavelength and reflectivity value) and the most relevant ones are assigned to their corresponding of the other spectra when their error distances are low. An example of the geometrical processing is depicted in Fig. 1 .

After matching the most relevant peaks, three key values are weighted to obtain the final error value: the ratio of unmatched peaks, the Euclidean distance between the matched peaks (for lambda and reflectivity axes) and the overlapped area between the two spectra.

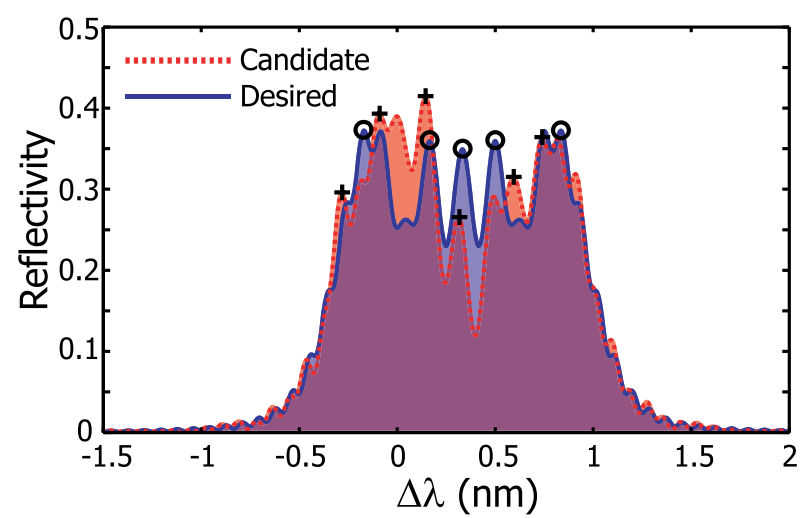

Fig. 1. Spectrum of a uniform FBG under the desired perturbation (solid line) is compared to the corresponding spectrum of a PSO candidate vector (dotted line). The error is computed using the main peaks of both spectra (crosses and circles) and the overlap under both curves. 
When the target (desired) spectrum has enough peaks to evaluate, the two first values are usually dominant in the error. However, for smoother spectra (i.e., a uniform strain profile applied to a uniform FBG has a single peak) the fine error value is given by the overlapped area under both curves, offering the required adaptability.

The described error metric has been employed as the quality measurement for the PSO algorithm. In order to improve the convergence, a smoothing process is applied to each of the PSO candidate strain profiles where each strain value of a candidate profile is averaged with its two neighbors (80\% itself and 10\% each neighbor). This step speeds the convergence up because the strain profiles to be reconstructed are usually continuous, thus each strain value is related to its neighbors (as happens in real measurements).

Several artificial spectra have been generated by applying nonuniform strain profiles to a uniform FBG of $10 \mathrm{~mm}$ length. These spectra have been employed to evaluate the synthetic algorithm performance.

A simulated spectrum obtained by applying a nonhomogeneous strain distribution to a uniform FBG has been analyzed. Two different spatial resolutions have been employed: 10 and 12 sections over $10 \mathrm{~mm}$ length. The results are depicted in Fig. 2 (top). The averaged values of five algorithm outputs for both resolutions (dashed line for $N=10$ and dotted line for $N=12$ ) are compared to the original strain distribution (solid line). Five individual outputs of the algorithm for the $N=12$ case are also detailed in Fig. 2 (top). The desired spectrum (solid line) is also compared to the best achieved solutions for both spatial resolutions in Fig. 2 (bottom) (dashed line for $N=10$ and dotted line for $N=12$ ). As the spatial resolution is increased (by
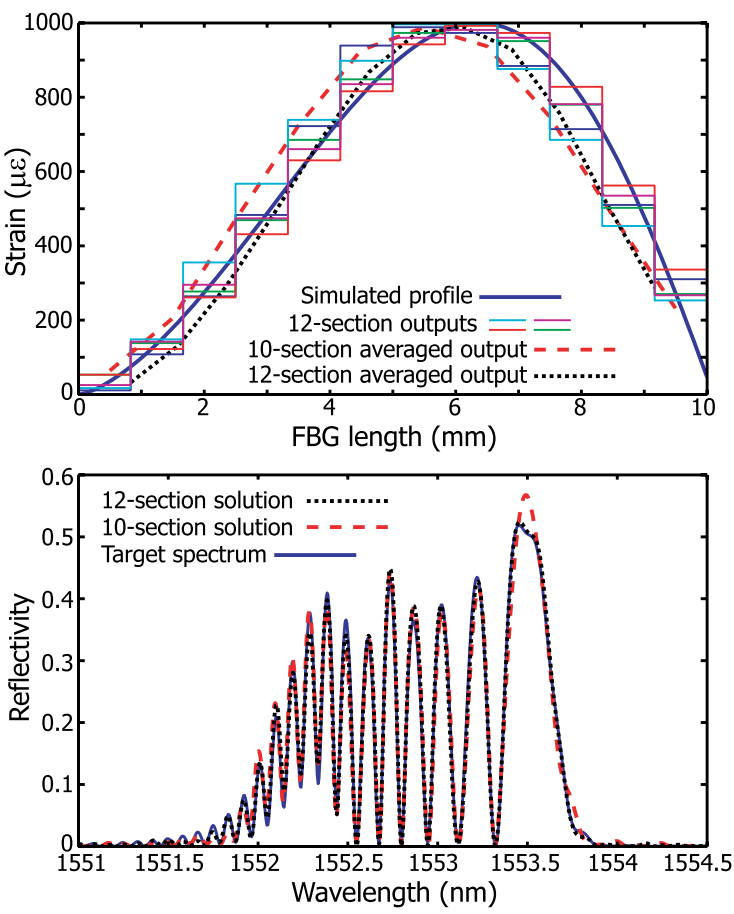

Fig. 2. Original strain profile is compared to PSO outputs for two different spatial resolutions (top). Best spectra for both resolutions are compared to the desired spectrum (bottom). increasing the number of sections, $N$ ), the achieved results are more accurate (mean strain error of $74.3 \mu \mathrm{\epsilon}$ for $N=10$ and of $25.8 \mu \epsilon$ for $N=12$ ), which can be noticed by analyzing the output spectra. Despite the error on the recovered strain profiles is higher than the synthetic spectra error, achieved strain resolution should be enough for mechanical sensing applications.

Due to the symmetry of uniform FBGs, there is an uncertainty about the direction of the perturbation: the spectra corresponding to an axial perturbation applied forward and backward are the same. This problem can be avoided by applying some apodizing function to the FBG structure instead of employing a uniform envelope. Otherwise, some a priori knowledge about the perturbation is required to resolve the uncertainty.

The geometrical processing scheme has also been tested using real data: a uniform FBG of $5 \mathrm{~mm}$ length has been written into standard optical fiber and it has been stressed using an asymmetric thermal perturbation. The setup is depicted in Fig. 3 and it is based on a Peltier heater. Part of the FBG is exposed to the air temperature while the other part is immersed into a thermal paste that is in direct contact to the Peltier heater. The heat transfer is more efficient within the thermal paste than through the air, creating an axial thermal difference in the FBG.

The Peltier temperature has been risen up to $70^{\circ} \mathrm{C}$ while room temperature was $25^{\circ} \mathrm{C}$, creating a nonuniform axial thermal distribution along the fiber, and consequently changing the spectral response of the FBG. The FBG spectrum has been captured using an optical spectrum analyzer (OSA, HP86140A) and a broadband light source (HP83437A) connected through a circulator. A synthetic spectrum (dashed line) based on an $N=10$ sections approach is compared to the measured (solid line) in Fig. 4 (top). Considering an FBG thermal sensitivity of $11 \mathrm{pm} /{ }^{\circ} \mathrm{C}$, the PSO outputs have been scaled to obtain the thermal perturbation along the FBG. The achieved results are detailed in Fig. $\underline{4}$ (bottom).

In Fig. 4, 10 of the PSO outputs are depicted (solid lines). These outputs have been averaged obtaining the axial thermal distribution along the FBG (dotted line). Analyzing the achieved results, the five initial sections exhibit a higher temperature because they are immersed into the thermal paste and, besides, the first section exhibits a higher temperature, being the closest to the Peltier heater. The last five sections show a decreasing temperature that corresponds to the cooling process of the air-surrounded fiber. For the measured spectrum, several runs have been performed obtaining an averaged standard deviation of less than $0.9^{\circ} \mathrm{C}$ with a maximum drift of $9^{\circ} \mathrm{C}$. Based on the simulated results and comparing the measured spectrum to the synthetic, it can be

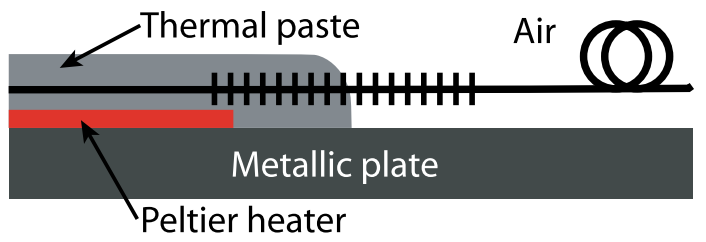

Fig. 3. Part of the FBG is immersed in thermal paste while the rest is exposed to the air. The temperature increase is provoked by a Peltier heater. 

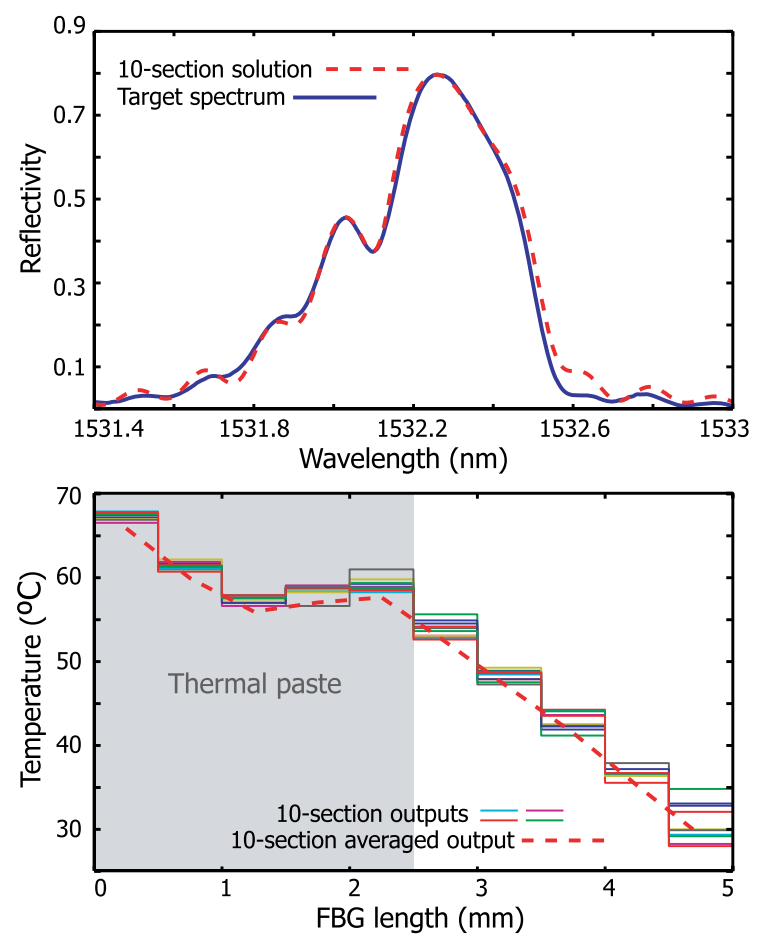

Fig. 4. Measured and a synthetic spectra obtained from a PSO output (top). Different PSO outputs and their averaged value (bottom).

seen that the geometrical processing is working properly as error metric by driving the PSO algorithm to the right solution. Despite typical problems of comparing measured to simulated spectra, slight differences between the simulated and the manufactured FBG structure, or lack of resolution of the measuring equipments $(60 \mathrm{pm}$ of the employed OSA), the achieved PSO outputs produce spectra very similar to the measured one, exhibiting a very high agreement to the employed setup.

Due to the iterative operation of PSO, obtaining the optimal solution cannot be ensured because it may fall into a local minimum. However, the convergence process can be evaluated with the error metric, guaranteeing a convergence range where the solution must fall within. When the final error value is higher than a threshold, PSO must be rerun with a greater number of swarm particles. If the sensitivity must be increased, several PSO outputs can be averaged to improve the results; however, this gain requires more PSO runs, thus the overall computation time increases. Another advantage of the proposed technique lies in the fact that the resolution is not limited by the measured data but by the computation time. The PSO algorithm performs a blind optimization over an $N$-dimensional problem, being $N$ the number of sections, thus the computation complexity increases exponentially with the number of sections.

In summary, a new processing scheme based on a comparison metric (geometrical processing) and a blind optimization technique (PSO) to resolve any axial perturbation applied to an FBG has been proposed and demonstrated. Simulated results show a good agreement with experiments, which in turn, correspond with the expected perturbations applied to the FBG using the proposed experimental setup. Simulated strain profiles and an experimental thermal profile have been recovered just from the intensity of reflection spectra. The achieved spatial resolution for the measured spectra was $\Delta \approx 0.5 \mathrm{~mm}$, being mainly limited by the computation time.

This work has been supported by the project TEC201020224-C02-02 and grant AP2009-1403. Special thanks to Dr. Mirapeix Serrano for his support and reviews and to Roberto Perez Sierra for his valuable collaboration with the experimental works.

\section{References}

1. J. Lopez-Higuera, Handbook of Optical Fibre Sensing Technology (Wiley, 2002).

2. Y. Rao, D. Jackson, L. Zhang, and I. Bennion, Opt. Lett. 21, 683 (1996).

3. J. Azana and M. Muriel, Opt. Lett. 25, 698 (2000).

4. X. Chapeleau, P. Casari, D. Leduc, Y. Scudeller, C. Lupi, R. Ny, and C. Boisrobert, J. Opt. A 8, 775 (2006).

5. M. LeBlanc, S. Huang, M. Ohn, A. Guemes, and A. Othonos, Opt. Lett. 21, 1405 (1996).

6. F. Casagrande, P. Crespi, A. Grassi, A. Lulli, R. Kenny, and M. Whelan, Appl. Opt. 41, 5238 (2002).

7. C. Cheng, Y. Lo, W. Li, C. Kuo, and H. Cheng, Appl. Opt. 46, 4555 (2007).

8. L. Rodriguez-Cobo, A. Cobo, and J. Lopez-Higuera, Opt. Express 20, 22429 (2012).

9. J. Kennedy and R. Eberhart, in Proceedings of IEEE International Conference on Neural Networks (IEEE, 1995), pp. 1942-1948.

10. J. Kennedy and R. Eberhart, Swarm Intelligence (Morgan Kaufmann, 2001).

11. R. Kashyap, Fiber Bragg Gratings (Academic, 1999). 